\title{
Anti-HER2 Therapy Beyond Second-Line for HER2- Positive Metastatic Breast Cancer: A Short Review and Recommendations for Several Clinical Scenarios from a Spanish Expert Panel
}

Noelia Martínez-Jañez ${ }^{a} \quad$ Ignacio Chacón $^{b} \quad$ Ana de Juan ${ }^{c} \quad$ Luis Cruz-Merino $^{d}$ Sònia del Barco $^{\mathrm{e}}$ Isaura Fernández ${ }^{\mathrm{f}}$ Paula García-Teijido ${ }^{g} \quad$ Amalia Gómez-Bernal $^{\mathrm{h}}$ Arrate Plazaola ${ }^{i}$ José Ponce ${ }^{j}$ Sonia Servitjak ${ }^{k}$ Pilar Zamoral

${ }^{a}$ Medical Oncology Department, Hospital Ramón y Cajal, Madrid, Spain;

${ }^{b}$ Medical Oncology Department, Hospital Virgen de la Salud, Toledo, Spain;

${ }^{\mathrm{c}}$ Medical Oncology Department, Hospital Universitario Marqués de Valdecilla, Santander, Spain;

${ }^{d}$ Medical Oncology Department, Hospital Virgen de la Macarena, Sevilla, Spain;

e Instituto Catalán de Oncología, Hospital Universitario Doctor Josep Trueta, Gerona, Spain;

${ }^{f}$ Hospital Xeral Cies de Vigo, Pontevedra, Spain;

${ }^{g}$ Hospital de Avilés, Asturias, Spain;

h Hospital Clínico de Salamanca, Salamanca, Spain;

i Onkologikoa, San Sebastián, Spain;

j Hospital General Universitario de Alicante, Alicante, Spain;

${ }^{k}$ Hospital del Mar, Barcelona, Spain;

' Hospital la Paz, Madrid, Spain

\section{Keywords}

Anti-HER2 - Metastatic breast cancer - Clinical practice

\section{Summary}

Background: The aim of this project was to provide an expert opinion regarding anti-human epidermal growth factor receptor 2 (HER2) therapy beyond second-line treatment of metastatic breast cancer (mBC). Methods: A group of experts discussed specific issues concerning anti-HER2 therapy in late-line settings in $\mathrm{mBC}$. Results: Trastuzumab emtansine (T-DM1) or dual HER2 blockade appeared to be good options for HER2-positive mBC after $\geq 2$ HER2-targeted therapies. Once an objective response has been achieved with anti-HER2-containing therapy, the anti-HER2 agent can be continued until progression of the disease, unacceptable toxicity or patient decision. $\mathrm{mBC}$ treated with $\geq 3$ consecutive lines of anti-HER therapy, $\geq 1$ being a dual HER2 blockade and with early progression of disease during a fourth or later-line treatment, are clinically resistant to anti-HER therapy. For progression of metastasis in the brain after anti-HER2 therapy, lapatinib and chemotherapy appear to be a good alternative after best local treatment. Conclusions: Further clinical trials are needed to provide valuable knowledge about the best treatment options in the later settings of $\mathrm{mBC}$.

(C) 2016 S. Karger GmbH, Freiburg

\section{Introduction}

During the past decade, clinical research has provided new targeted treatments for breast cancer that have changed the prognosis and clinical management of this malignancy. In particular, patients with metastatic breast cancer $(\mathrm{mBC})$ and the overexpression of human epidermal growth factor receptor 2 (HER2) have experienced a remarkable improvement in terms of overall survival (OS) in the last 10 years [1]. This subtype is found in $15-20 \%$ of patients with $\mathrm{mBC}$ and was associated with poorer prognosis and shorter OS before the introduction of the anti-HER2 agent trastuzumab into clinical practice $[1,2]$.

\section{KARGER}

Fax +497614520714

\section{(c) 2016 S. Karger GmbH, Freiburg}

1661-3791/16/0112-0133\$39.50/0 
There are 2 families of HER2-targeting drugs: monoclonal antibodies and tyrosine kinase inhibitors. Trastuzumab was the first anti-HER2 monoclonal antibody available for the treatment of mBC. Pertuzumab, a monoclonal antibody that inhibits HER2 dimerization, was authorized by the European Medicine Agency (EMA) in March 2013 for the first-line treatment of $\mathrm{mBC}$ combined with docetaxel and trastuzumab [3]. More recently, in November of 2013, the European Commission granted a marketing authorization for trastuzumab emtansine (T-DM1), an antibody-drug conjugate that contains 2 active components: trastuzumab and an anti-tubulin agent, emtansine. T-DM1 is approved for the second-line treatment of HER2- positive $\mathrm{mBC}$, previously treated with trastuzumab and/ or taxanes, separately or in combination [4]. Lapatinib is currently the first and only tyrosine kinase inhibitor available for $\mathrm{mBC}$. Neratinib and afatinib are currently under evaluation in clinical trials. Based on pretreatment, hormone receptor expression and patient characteristics, anti-HER2 therapy can be combined with chemotherapy, endocrine therapy, or other anti-HER2 agents [5].

Clinical guidelines recommend the use of anti-HER2-based regimens for the first and second-line treatment of HER-positive $\mathrm{mBC}$, but there is no consensus about treatment following secondline therapy, particularly regarding the use of an HER2 blockade. It is also unclear when to stop anti-HER2 therapy and how to define resistance to anti-HER therapy $[2,6]$. Furthermore, novel antiHER2 therapies for $\mathrm{mBC}$, such as pertuzumab or T-DM1, will add new alternatives for treatment decision-making.

Given the lack of consensus regarding the use of anti-HER2 therapy beyond the second-line treatment of HER2-positive mBC and the fact that most of these patients usually receive more than 2-3 lines of treatment, a group of Spanish experts was established to discuss the state of anti-HER2 therapy. This project was a clinicians' initiative with the purpose of seeking recommendations for the frequent situations in clinical practice for which there is not enough supporting evidence from the scientific literature. Therefore, the aim of this project was to provide a review on treatment recommendations for HER2 $\mathrm{mBC}$ patients, to discuss specific clinical scenarios after second-line treatment for which there is no quantitative research and to suggest an algorithm for the management of HER2-positive mBC.

\section{Methods}

A group of 12 medical oncologists specializing in breast cancer were invited to discuss the literature and their clinical experience on the topic of anti-HER2 therapy in mBC. 2 members of the group were in charge of coordinating the discussions and the information provided before and after the meetings.

To address the questions not answered by the available evidence, several clinical scenarios were proposed: (1) What to do when the disease progresses after a second-line of trastuzumab-containing regimen? (2) When to stop considering anti-HER2 therapy as an alternative? (3) When to re-treat with trastuzumab after a lapatinib-containing regimen? (4) What to do in patients with brain metastasis with regard to anti-HER2 therapy? (5) When to continue trastuzumab after a complete response?

The group of experts discussed these scenarios in 2 rounds. In the first round, the participants met and established what to do in the clinical scenarios

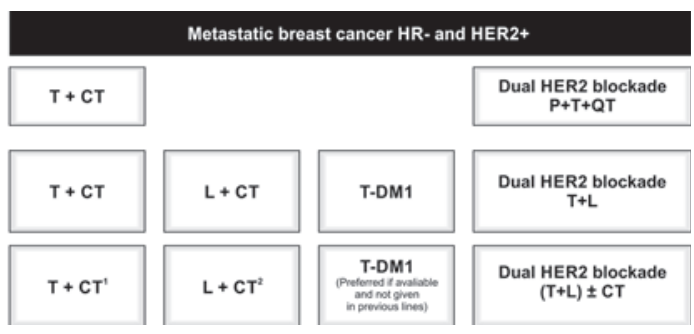

Fig. 1. Anti-HER2 therapy for HER2-positive metastatic breast cancer. After complete response with an anti-HER2-based therapy, in the absence of any evidence pointing to a necessity to discontinue anti-HER2, continuation of anti-HER2 therapy could be contemplated, until a possible progression of disease, unacceptable toxicity or patient decision. Resistance: Patients with metastatic breast cancer ( $\mathrm{mBC}$ ) meeting all of the following criteria can be considered as patients with resistance to anti-HER2. There are at least 3 lines of antiHER2 therapy, and at least 1 dual anti-HER2-targeted therapy that can be used to treat progression of disease after the first assessment of the tumor. CT = chemotherapy; ET = endocrine therapy; $\mathrm{HR}=$ hormone receptor; $\mathrm{L}=$ lapatinib; $\mathrm{P}=$ pertuzumab; $\mathrm{PR}=$ progesterone receptor; $\mathrm{T}=$ trastuzumab; $\mathrm{T}-\mathrm{DM} 1=$ tras tuzumab emtansine.

of interest based on the scientific evidence and their clinical experience. The coordinators compiled the comments of all of the participants and proposed a first draft of recommendations for each scenario. In the second round, the participants declared their agreement or disagreement with the drafted recommendations and gave further comments to this proposal.

The group coordinators compiled the comments received and wrote a second draft that was sent to all of the participants. This draft was reviewed and modified until the agreement of all of the participants was achieved. Finally, the group of experts proposed a treatment-algorithm for HER2-positive mBC incorporating these clinical scenarios.

In this paper, we first summarize the available literature and then provide recommendations for various clinical scenarios and a treatment algorithm that goes beyond the second line.

\section{Anti-HER-2 Therapy for HER2-Positive $\mathrm{mBC}$ in the First-Line Setting}

The availability of anti-HER2 therapy has changed the treatment paradigm of $\mathrm{mBC}$. Starting with the first-line setting, the following 2 subsets among HER-positive $\mathrm{mBC}$ exist: those that are hormone receptor (HR) negative and those that are HR-positive. For HER2-positive and HR-negative cases, anti-HER2 treatment may be added to the first-line standard chemotherapy (fig. 1) [2, 6]. In a phase III clinical trial aiming to demonstrate a significant improvement in terms of progression-free survival (PFS) when trastuzumab was added to standard chemotherapy (anthracycline plus cyclophosphamide or paclitaxel) for HER2-positive mBC, clinically relevant results in PFS in favor of adding trastuzumab to either chemotherapy were obtained (hazard ratio (HR) 0.51; 95\% confidence interval (CI) 0.41-0.63)) [7]. In another phase III clinical trial in patients with HER2-positive $\mathrm{mBC}$, lapatinib plus paclitaxel (compared with lapatinib placebo plus paclitaxel) significantly improved OS (HR 0.74; 95\% CI 0.58-0.94) and PFS (HR 0.52 ; 95\% CI 0.42-0.64) [8]. However, when comparing both alternatives, trastuzumab and lapatinib added to taxane-based chemo- 
Table 1. Randomized phase III trials of secondline treatments for HER2-positive metastatic breast cancer

\begin{tabular}{|c|c|c|c|}
\hline Reference (trial identification) & Treatment (n) & Prior treatment, $\mathrm{n}(\%)$ & HR (95\% CI) \\
\hline $\begin{array}{c}\text { von Minckwitz, } 2009 \text { [21] } \\
\text { von Minckwitz, } 2011 \text { [22] } \\
\text { (GBG 26/BIG 03-05) }\end{array}$ & $\begin{array}{l}\mathrm{T}+\mathrm{C}(78) \\
\mathrm{C}(\mathrm{n}=78)\end{array}$ & $\begin{array}{l}>24 \text { months prior T, } 57(81) \\
>24 \text { months prior T, } 61(79)\end{array}$ & $\begin{array}{l}\text { TTP: } 0.69(0.48-0.97)^{*}, \mathrm{p}=0.0338 \\
\text { OS: } 0.94(0.65-1.3), \mathrm{p}=0.73\end{array}$ \\
\hline $\begin{array}{l}\text { Cameron, } 2008[24] \\
\text { Cameron, } 2010[25]\end{array}$ & $\begin{array}{l}\mathrm{L}+\mathrm{C}(207) \\
\mathrm{C}(201)\end{array}$ & $\begin{array}{l}\geq 3 \text { CT regimens, } 176(85) \\
>8 \text { weeks prior T, } 83(40) \\
\geq 3 \text { CT regimens, } 164(82) \\
>8 \text { weeks prior T, } 77(38)\end{array}$ & $\begin{array}{l}\text { TTP: } 0.57(0.43-0.77)^{*}, \mathrm{p}<0.001 \\
\text { OS: } 0.87(0.71-1.08), \mathrm{p}=0.210\end{array}$ \\
\hline $\begin{array}{l}\text { Verma, } 2012[28] \\
\quad(\text { EMILIA trial) }\end{array}$ & $\begin{array}{l}\text { T-DM1 (495) } \\
\mathrm{L}+\mathrm{C}(496)\end{array}$ & $\begin{array}{l}>1 \mathrm{CT} \text { line of } \mathrm{mBC}, 191(39) \\
\text { prior } \mathrm{T} \text { for } \mathrm{mBC}, 417(84) \\
>1 \mathrm{CT} \text { line of } \mathrm{mBC}, 191(39) \\
\text { prior } \mathrm{T} \text { for } \mathrm{mBC}, 419(84)\end{array}$ & $\begin{array}{l}\text { PFS: } 0.65(0.55-0.77)^{*}, \mathrm{p}<0.001 \\
\text { OS: } 0.68(0.55-0.85), \mathrm{p}<0.001\end{array}$ \\
\hline $\begin{array}{l}\text { Blackwell, } 2012 \text { [29] } \\
\text { (EGF104900) }\end{array}$ & $\mathrm{L}+\mathrm{T}(148)$ & $\begin{array}{l}\geq 6 \mathrm{CT} \text { regimens, } 50(34) \\
\text { median no. of prior } \mathrm{T} \text { regimens } \\
\text { for } \mathrm{mBC}, 3 \\
\text { median days from last } \mathrm{T}=27 \\
\geq 6 \mathrm{CT} \text { regimens, } 41(28) \\
\text { median no. of prior } \mathrm{T} \text { regimens } \\
\text { for } \mathrm{mBC}, 3 \\
\text { median days from last } \mathrm{T}=25\end{array}$ & $\begin{array}{l}\text { PFS: } 0.74(0.58-0.94)^{*}, \mathrm{p}=0.011 \\
\text { OS: } 0.74(0.57-0.97), \mathrm{p}=0.026\end{array}$ \\
\hline
\end{tabular}

(*) Primary analysis.

$\mathrm{T}=$ trastuzumab, $\mathrm{C}$ = capecitabine, $\mathrm{L}=$ lapatinib, $\mathrm{CT}=$ chemotherapy, $\mathrm{HR}$ = hazard ratio, $\mathrm{CI}=$ confidence interval, $\mathrm{mBC}=$ metastatic breast cancer, $\mathrm{OS}=$ overall survival, $\mathrm{PFS}=$ progression-free survival, $\mathrm{TTP}=$ time to progression. therapy, lapatinib led to inferior PFS compared to trastuzumab (HR 1.33; 95\% CI 1.06-1.67) [9]. Other cytotoxic agents, such as vinorelbine, platinum compounds and gemcitabine, have also shown clinical benefit when administered in combination with trastuzumab for the first-line treatment of HER2-positive $\mathrm{mBC}$ [10-12].

In the clinical phase III CLEOPATRA trial, the combination of docetaxel with a dual HER2 blockade with pertuzumab and trastuzumab showed a significant benefit in PFS and OS compared to the former treatment of standard trastuzumab plus docetaxel (HR 0.69; 95\% CI 0.58-0.81) and OS (HR 0.66; 95\% CI 0.52-0.84). [13]. Based on these results, the American Society of Clinical Oncology (ASCO) recommends the combination of trastuzumab, pertuzumab and taxane for first-line treatment, unless the patient has a contraindication to taxanes [14]. The new combined drug T-MD1 has also shown a significant improvement in PFS when compared with trastuzumab plus docetaxel as a first-line treatment for HER2postive $\mathrm{mBC}$ [15], although it has not yet been recommended for a first-line treatment of $\mathrm{mBC}$ (fig. 1).

Similarly, for patients with HR-positive and HER2-positive $\mathrm{mBC}$, addition of anti-HER2 therapy to first-line endocrine therapy has also been recommended, based on the significant improvement of PFS observed in phase III trials when lapatinib was added to letrozole $[16,17]$ or when trastuzumab was added to anastrozole $[18,19]$; for a small subset of HR-positive patients, endocrine therapy alone seems to be sufficient treatment. When chemotherapy is indicated as a first-line treatment in this subset of patients, treatment decision making is performed as described above for HRnegative $\mathrm{mBC}$ overexpressing HER2 (fig. 1).
Special consideration is required for those patients with HER2positive $\mathrm{mBC}$ who experience progression of the disease during or after adjuvant trastuzumab, for whom the standard of care has not been well-established [20]. Patient characteristics, tumor biology, prior treatments and potential resistance to trastuzumab should be considered for trastuzumab treatment selection. The ASCO practice guideline suggests following a first-line approach when recurrence is observed after 12 months from when the patient finished the trastuzumab-based adjuvant therapy, and to follow a secondline approach when recurrence is observed within 12 months following trastuzumab-based adjuvant therapy [14]. In this context, the EMA has recently authorized T-DM1 for the treatment of HER2-positive patients with recurrence of disease during or within 6 months of completing adjuvant therapy [4].

\section{Anti-HER-2 Therapy for HER2-Positive $\mathrm{mBC}$ in the Second-Line Setting}

In the second-line setting, continuing with the HER2 blockade is the recommended approach [14]. To do so, there are 3 main strategies: adding trastuzumab or lapatinib to chemotherapy (same as in the first-line setting), treatment with T-DM1, or applying a dual HER2 blockade approach (i.e. treatment with lapatinib plus trastuzumab) (fig. 1). The phase III trials supporting these recommendations are summarized in table 1. A German clinical trial, whose principal objective was to demonstrate a benefit in time to progression (TTP) when adding trastuzumab to capecitabine, was prematurely closed due to poor accrual [21]. However, a significant 
Table 2. Clinical scenarios

Scenario 1. What to do when the disease progresses after a second-line of trastuzumab containing-regimen?

Background: There is a substantial lack of evidence on what to do beyond a second-line trastuzumab-containing regimen. However, the clinical trial TH3RESA showed promising results with T-DM1. In this setting, T-DM1 provided a significant improvement in PFS (HR=0.53, 95\% CI 0.42-0.66) with a better safety profile than the physician's choice treatment in patients with $\mathrm{mBC}$ treated with at least 2 previous lines of anti-HER2 therapy [34]. In addition, the combination of trastuzumab and lapatinib has been shown to improve OS in heavily pretreated patients with disease progression after adjuvant or metastatic treatment [29, 35]. Recommendation: HER2-positive $\mathrm{mBC}$ with progression of disease after 2 or more previous HER2-targeted therapies can be treated with T-DM1 or dual HER2 blockade. Whenever T-DM1 is available and was not given in previous lines, it seems to be the best treatment option in almost all settings. Only when lapatinib is a particularly attractive option (e.g. if there are brain metastases; see Scenario 4) may the dual HER2 blockade be more promising than T-DM1.

Scenario 2. When to consider clinical resistance to anti-HER2 therapy?

Background: This scenario refers to the concept of a clinical resistance to anti-HER2 rather than a molecular resistance to anti-HER2 described elsewhere [36] It is based on the clinical experience of the group of experts in situations where there is lack of clinical benefit.

Recommendation: Patients with $\mathrm{mBC}$ meeting the 3 following criteria can be considered to possess clinical resistance to anti-HER2:

At least 3 consecutive lines of anti-HER2 therapy

At least 1 dual anti-HER 2 targeted therapy

Progression of the disease after the first assessment of tumor during fourth- or later-line treatment

Scenario 3. When to re-treat with trastuzumab after lapatinib-containing regimen?

Background: Re-treatment with trastuzumab after disease progression during lapatinib-containing therapy has been shown to provide a clinical benefit of $47 \%$ [37]. Even after exposure to T-DM1, re-treatment with trastuzumab appears to have a potential benefit [38].

Recommendation: For those patients with $\mathrm{mBC}$ and progression of the disease after a lapatinib-containing second-line regimen, re-treatment with trastuzumab can be considered based on the following criteria:

T-DM1 (if available, and not given in previous lines) may have priority over the others

Dual HER2 blockade

Added to chemotherapy

Scenario 4 . What to do in patients with brain metastases concerning anti-HER2 therapy?

Background: Anti-HER2 therapies have limited potential to cross the blood-brain barrier, and the available evidence is not strong enough to recommend treatment for these patients [39]. ASCO practice guidelines suggest the continuation of HER2-targeted therapy for patients who receive standard therapy for the treatment of brain metastases (surgical and/or radiotherapy) and whose systemic disease is progressive at the time of brain metastasis diagnosis [19]. For systemic therapy, lapatinib may be a potential option in this setting, although the evidence so far is rather exploratory [40]. Recently, the drug penetration of capecitabine and lapatinib uptake in resected brain metastases from women with metastatic breast cancer has been reported in a case-series study [41]. Therefore, the combination of lapatinib and capecitabine may be considered in this setting [19].

Recommendation: For patients with $\mathrm{mBC}$ and brain metastases with progression of disease after anti-HER2 therapy, the combination of lapatinib and chemotherapy may be a good option after best local treatment.

Scenario 5. When to continue trastuzumab after a complete response?

Background: If there is evidence supporting the use of trastuzumab beyond the progression of disease [22, 42], it is recommended to continue with trastuzumab while there is a clinical benefit [14].

Recommendation: After a complete response with an anti-HER2-containing therapy, anti-HER2 therapy can be continued until the progression of disease, unacceptable toxicity or patient decision, in the absence of evidence to discontinue anti-HER2 earlier.

Patients' preferences should be discussed to make a patient-physician shared decision.

T-DM1 = trastuzumab emtansine, $\mathrm{PFS}=$ progression-free survival, $\mathrm{HR}=$ hazard ratio, $\mathrm{CI}=$ confidence interval, $\mathrm{mBC}=$ metastatic breast cancer, $\mathrm{HER} 2=$ human epidermal growth factor receptor 2, OS = overall survival, ASCO = American Society of Clinical Oncology.

improvement in TTP could be demonstrated, although a significant improvement in OS was not observed (table 1) [21, 22]. The low rate of recruitment is likely to be explained by the US Food and Drug Administration approval of lapatinib in combination with capecitabine that was granted at that time based on the results of another phase III clinical trial [23]. The enrollment in this trial with lapatinib was also stopped prematurely based on the superiority of TTP observed in the lapatinib plus capecitabine group in the interim analysis [23]. Subsequent analyses showed similar benefits in TTP [24], although no differences in OS between the combined therapy group and the capecitabine monotherapy group were observed [25]. However, when accounting for treatment crossover (35 patients randomly assigned to the capecitabine monotherapy group crossed over to combination therapy when enrollment into the study was prematurely closed), a statistically significant improvement in OS was obtained in favor of the lapatinib plus capecitabine group (HR 0.80; 95\% CI, 0.64-0.99) [25].

The clinical benefit observed with T-DM1 for HER2-positive $\mathrm{mBC}$ in phase II clinical trials $[26,27]$ was confirmed in a phase III trial in which T-DM1 was compared with lapatinib plus capecit- 
abine in patients with $\mathrm{mBC}$ previously treated with trastuzumab and a taxane [28]. In this phase III trial, PFS and OS improved significantly with T-DM1 treatment compared to lapatinib plus capecitabine (table 1) [28].

The third strategy recommended for the second-line treatment of $\mathrm{mBC}$ is the HER2 dual blockade using lapatinib in combination with trastuzumab (fig. 1). This strategy is based on the results obtained in the EGF104900 phase III clinical trial in which lapatinib plus trastuzumab showed a significant benefit in PFS and OS compared to lapatinib monotherapy in patients with HER2-positive $\mathrm{mBC}$ previously treated with trastuzumab-containing regimens [29].

Finally, although not yet approved for second-line treatment, pertuzumab in combination with trastuzumab has shown an objective response rate of $24.2 \%$ with a median PFS of 5.5 months and a good safety profile in a phase II single arm trial of patients with HER2-positive $\mathrm{mBC}$ pre-treated with a prior trastuzumab-containing regimen [30].

\section{Beyond the Second-Line of Anti-HER2 Therapy}

Beyond second-line treatment, there is little evidence on how patients should be treated. Although there is some consensus on continuing with the HER2 blockade [14], clinical guidelines are ambiguous on how many lines of anti-HER2 therapy may be acceptable and whether HER2 blockade should be continued long term [2]. Furthermore, novel anti-HER2 drugs that are about to become available or have been recently authorized could complicate treatment decision making in advanced settings. For example, there is strong evidence for the use of T-DM1 after a second or greater HER2-targeted treatment for patients who have not received it before [14]. However, it is not clear what should be done for patients who have been previously treated with T-DM1. Some authors have suggested that pertuzumab could be an alternative in this setting for patients not previously exposed to pertuzumab [19] Pertuzumab and trastuzumab should be combined with chemotherapy (e.g. vinorelbine) in this setting.

We specifically defined 5 clinical scenarios for which controversy may exist on what to do with regard to anti-HER2 therapy beyond the second-line of anti-HER2 therapy. The background and agreed recommendations for these 5 scenarios are summarized in table 2. When available, the recommendations are supported with scientific evidence. However, for most of the scenarios, the consensus opinion of the group of experts is provided given the lack of scientific evidence. The treatment algorithm proposed that incorporates these recommendations is provided in figure 1.

The safety of anti-HER2 drugs is a key issue in treatment decision making. Beyond the second-line, it is particularly relevant to account for potential long-term adverse reactions, such as cardiotoxicity. In this sense, trastuzumab-related cardiac events appear to be reversible and unrelated to the cumulative dose, with re-challenge generally well tolerated [31]. However, for high-risk populations, such as elderly patients with cardiovascular disease and/or diabetes, it would be advisable to consider continuous cardiac monitoring [32]. There is less concern about cardiotoxicity when administering new anti-HER2 drugs. In particular, T-DM1 appears to be remarkably well tolerated in patients with $\mathrm{mBC}$ [33].

\section{Conclusions}

The introduction of anti-HER2 drugs has changed the treatment and prognosis of the subset of patients with $\mathrm{mBC}$ overexpressing HER2, a subgroup that was previously associated with poorer outcomes. Novel anti-HER2 drugs provide new alternatives that are likely to improve the prognosis of these patients to an even greater extent. During the past decade, the standard treatment approach has been to combine anti-HER2 drugs with the indicated therapy (either chemotherapy or hormonotherapy). With the new anti-HER drugs, dual HER2 blockade tends to be a good alternative for these patients. T-DM1, a new conjugate that combines targeted therapy and a cytotoxic drug, has shown significant results in efficacy and a remarkably good safety profile in patients with $\mathrm{mBC}$.

Although the clinical trial TH3RESA [34] provides good evidence on what to do after the second-line setting concerning antiHER2 therapy in patients with $\mathrm{mBC}$, there is still a substantial lack of evidence on how to treat patients previously treated with T-DM1 and what to do beyond second-line treatment.

Further clinical trials could answer some of the questions that arise in the third-line treatment and thereafter. Nevertheless, there will always be a lack of evidence-based knowledge on what to do in late-line settings, particularly in those patients who are usually excluded from clinical trials due to ethical and/or safety reasons. In those situations, where there is a lack of applicable evidence, individual patient management requires making well-balanced decisions between the available evidence, clinical judgment and patient preferences.

\section{Acknowledgements}

The authors wish to thank Teresa Hernando from Cociente S.L. (Madrid, Spain) for her support in preparing the first draft of this manuscript. Roche Pharmaceuticals funded the scientific meetings and medical writing services. The authors made all decisions about the final content of this manuscript. All authors have approved the final version of the submitted manuscript.

\section{Disclosure Statement}

All authors have received fees from Roche Farma for their participation in the submitted work. The authors declare the following potential conflict of interest not related with the submitted work: Dr. Martínez-Jañez, Dr. Chacón, Dr. de Juan, Dr. Gómez-Bernal, Dr. Fernández, Dr. Plazaola, Dr. Ponce, Dr. Servitja and Dr. Zamora declare no further conflicts of interest. Dr. Cruz-Merino has served as a speaker, a consultant and an advisory board member for Roche, Novartis, GlaxoSmithklineBeecham, Bristol Myers Squibb and Celgene and has received research funding from Roche; Dr. del Barco has participated in advisory boards of Celgene, Novartis y Astra Zeneca during the last year; Dr. García-Teijido has served as a speaker, a consultant and an advisory board member for Roche, Novartis, Astra Zeneca, Amgen and has received research funding from Eisai. 


\section{References}

1 Lobbezoo DJ, van Kampen RJ, Voogd AC, et al.: Prognosis of metastatic breast cancer subtypes: The hormone receptor/HER2-positive subtype is associated with the most favorable outcome. Breast Cancer Res Treat 2013;141:507-514.

2 Llombart Cussac A, de la Haba Rodriguez J, Ruiz Simon A, et al.: SEOM clinical guidelines for the management of metastatic breast cancer 2013. Clin Transl Oncol 2013;15:1004-1010.

3 Montalvo I, Ortega L, López X, et al.: Changes in prolactin levels and sexual function in young psychotic patients after switching from long-acting injectable risperidone to paliperidone palmitate. Int Clin Psychopharmacol 2013;28:46-49.

4 Lally J, Byrne F, Walsh E: A case of paliperidone-palmitate-induced tardive dyskinesia. Gen Hosp Psychiatry 2013;35:e5-e7.

5 Nielsen DL, Kumler I, Palshof JA, Andersson M: Efficacy of HER2-targeted therapy in metastatic breast cancer. Monoclonal antibodies and tyrosine kinase inhibitors. Breast 2013;22:1-12.

$6 \mathrm{NCCN}$ clinical practice guidelines in oncology (NCCN Guidelines) ${ }^{\circledR}$ breast cancer. Version 3, 2013.

7 Slamon DJ, Leyland-Jones B, Shak S, et al.: Use of chemotherapy plus a monoclonal antibody against HER2 for metastatic breast cancer that overexpresses HER2. N Engl J Med 2001;344:783-792.

8 Guan Z, Xu B, DeSilvio ML, et al.: Randomized trial of lapatinib versus placebo added to paclitaxel in the treatment of human epidermal growth factor receptor 2-overexpressing metastatic breast cancer. J Clin Oncol 2013;31:1947-1953.

9 Gelmon KA, Boyle F, Kaufman B, et al.: Open-label phase III randomized controlled trial comparing taxane-based chemotherapy (tax) with lapatinib (l) or trastuzumab ( $\mathrm{t}$ ) as first-line therapy for women with $\mathrm{HER} 2^{+}$metastatic breast cancer: Interim analysis (IA) of NCIC CTG MA.31/GSK EGF 108919. ASCO 2012 (abstract number LBA671),

10 Burstein HJ, Keshaviah A, Baron AD, et al.: Trastuzumab plus vinorelbine or taxane chemotherapy for HER2-overexpressing metastatic breast cancer: The trastuzumab and vinorelbine or taxane study. Cancer 2007;110:965-972.

11 Pegram MD, Pienkowski T, Northfelt DW, et al.: Results of two open-label, multicenter phase II studies of docetaxel, platinum salts, and trastuzumab in HER2positive advanced breast cancer. J Natl Cancer Inst 2004;96:759-769.

12 Yardley DA, Burris HA 3rd, Simons L, et al.: A phase II trial of gemcitabine/carboplatin with or without trastuzumab in the first-line treatment of patients with metastatic breast cancer. Clin Breast Cancer 2008;8: 425-431.

13 Swain SM, Kim SB, Cortes J, et al.: Pertuzumab, trastuzumab, and docetaxel for HER2-positive metastatic breast cancer (CLEOPATRA study): Overall survival results from a randomised, double-blind, placebo-controlled, phase 3 study. Lancet Oncol 2013;14:461-471.

14 Giordano SH, Temin S, Kirshner JJ, et al.: Systemic therapy for patients with advanced human epidermal growth factor receptor 2-positive breast cancer: American Society of Clinical Oncology clinical practice guideline. J Clin Oncol 2014;32:2078-2099.
5 Hurvitz SA, Dirix L, Kocsis J, et al.: Phase II randomized study of trastuzumab emtansine versus trastuzumab plus docetaxel in patients with human epidermal growth factor receptor 2-positive metastatic breast cancer. J Clin Oncol 2013;31:1157-1163.

16 Johnston S, Pippen J Jr, Pivot X, et al.: Lapatinib combined with letrozole versus letrozole and placebo as first-line therapy for postmenopausal hormone receptor-positive metastatic breast cancer. J Clin Oncol 2009;27:5538-5546.

17 Schwartzberg LS, Franco SX, Florance A, et al.: Lapatinib plus letrozole as first-line therapy for HER- $2^{+}$hormone receptor-positive metastatic breast cancer. Oncologist 2010;15:122-129.

18 Kaufman B, Mackey JR, Clemens MR, et al.: Trastuzumab plus anastrozole versus anastrozole alone for the treatment of postmenopausal women with human epidermal growth factor receptor 2-positive, hormone receptor-positive metastatic breast cancer: Results from the randomized phase III tandem study. J Clin Oncol 2009;27:5529-5537.

19 Ramakrishna N, Temin S, Chandarlapaty S, et al.: Recommendations on disease management for patients with advanced human epidermal growth factor receptor 2-positive breast cancer and brain metastases: American Society of Clinical Oncology clinical practice guideline. J Clin Oncol 2014;32:2100-2108.

20 De Mattos-Arruda L, Cortes J: Advances in first-line treatment for patients with HER- $2^{+}$metastatic breast cancer. Oncologist 2012;17:631-644.

21 von Minckwitz G, du Bois A, Schmidt M, et al.: Trastuzumab beyond progression in human epidermal growth factor receptor 2-positive advanced breast cancer: A German breast group 26/Breast International Group 03-05 study. J Clin Oncol 2009;27:1999-2006.

22 von Minckwitz G, Schwedler K, Schmidt M, et al.: Trastuzumab beyond progression: Overall survival analysis of the GBG 26/BIG 3-05 phase III study in HER2-positive breast cancer. Eur J Cancer 2011;47: 2273-2281.

23 Geyer CE, Forster J, Lindquist D, et al.: Lapatinib plus capecitabine for HER2-positive advanced breast cancer. N Engl J Med 2006;355:2733-2743.

24 Cameron D, Casey M, Press M, et al.: A phase III randomized comparison of lapatinib plus capecitabine versus capecitabine alone in women with advanced breast cancer that has progressed on trastuzumab: Updated efficacy and biomarker analyses. Breast Cancer Res Treat 2008;112:533-543.

25 Cameron D, Casey M, Oliva C, et al.: Lapatinib plus capecitabine in women with HER-2-positive advanced breast cancer: Final survival analysis of a phase III randomized trial. Oncologist 2010;15:924-934.

26 Krop IE, LoRusso P, Miller KD, et al.: A phase II study of trastuzumab emtansine in patients with human epidermal growth factor receptor 2-positive metastatic breast cancer who were previously treated with trastuzumab, lapatinib, an anthracycline, a taxane, and capecitabine. J Clin Oncol 2012;30:3234-3241.

27 Burris HA 3rd, Rugo HS, Vukelja SJ, et al.: Phase II study of the antibody drug conjugate trastuzumabDM1 for the treatment of human epidermal growth factor receptor 2 (HER2)-positive breast cancer after prior HER2-directed therapy. J Clin Oncol 2011;29: $398-405$.
28 Verma S, Miles D, Gianni L, et al.: Trastuzumab emtansine for HER2-positive advanced breast cancer. N Engl J Med 2012;367:1783-1791.

29 Blackwell KL, Burstein HJ, Storniolo AM, et al.: Overall survival benefit with lapatinib in combination with trastuzumab for patients with human epidermal growth factor receptor 2-positive metastatic breast cancer: Final results from the EGF104900 study. J Clin Oncol 2012;30:2585-2592.

30 Baselga J, Gelmon KA, Verma S, et al.: Phase II trial of pertuzumab and trastuzumab in patients with human epidermal growth factor receptor 2-positive metastatic breast cancer that progressed during prior trastuzumab therapy. J Clin Oncol 2010;28:1138-1144.

31 Ewer SM, Ewer MS: Cardiotoxicity profile of trastuzumab. Drug Saf 2008;31:459-467.

32 Serrano C, Cortes J, De Mattos-Arruda L, et al.: Trastuzumab-related cardiotoxicity in the elderly: A role for cardiovascular risk factors. Ann Oncol 2012;23: 897-902.

33 Sendur MA, Aksoy S, Altundag K: Cardiotoxicity of novel HER2-targeted therapies. Curr Med Res Opin 2013;29:1015-1024.

34 Krop IE, Kim SB, Gonzalez-Martin A, et al.: Trastuzumab emtansine versus treatment of physician's choice for pretreated HER2-positive advanced breast cancer (TH3RESA): A randomised, open-label, phase 3 trial. Lancet Oncol 2014;689-699.

35 Blackwell KL, Burstein HJ, Storniolo AM, et al.: Randomized study of lapatinib alone or in combination with trastuzumab in women with ErbB2-positive, trastuzumab-refractory metastatic breast cancer. J Clin Oncol 2010;28:1124-1130.

36 Bates M, Sperinde J, Kostler WJ, et al.: Identification of a subpopulation of metastatic breast cancer patients with very high HER2 expression levels and possible resistance to trastuzumab. Ann Oncol 2011;22:20142020.

37 Gori S, Montemurro F, Spazzapan S, et al.: Retreatment with trastuzumab-based therapy after disease progression following lapatinib in HER2-positive metastatic breast cancer. Ann Oncol 2012;23:1436-1441.

38 Olson EM, Lin NU, DiPiro PJ, et al.: Responses to subsequent anti-HER2 therapy after treatment with trastuzumab-DM1 in women with HER2-positive metastatic breast cancer. Ann Oncol 2012;23:93-97.

39 Larsen PB, Kumler I, Nielsen DL: A systematic review of trastuzumab and lapatinib in the treatment of women with brain metastases from HER2-positive breast cancer. Cancer Treat Rev 2013;39:720-727.

40 Iwata H, Narabayashi M, Ito Y, et al.: A phase II study of lapatinib for brain metastases in patients with HER2-overexpressing breast cancer following trastuzumab based systemic therapy and cranial radiotherapy: Subset analysis of Japanese patients. Int J Clin Oncol 2013;18:621-628.

41 Morikawa A, Peereboom D, Smith Q, et al.: Clinical evidence for drug penetration of capecitabine and lapatinib uptake in resected brain metastases from women with metastatic breast cancer. 2013 ASCO Annual Meeting. J Clin Oncol 2013;31(suppl):abstr 514.

42 Waddell T, Kotsori A, Constantinidou A, et al.: Trastuzumab beyond progression in HER2-positive advanced breast cancer: The Royal Marsden experience. Br J Cancer 2011;104:1675-1679. 